Cosmogenic production of tritium in dark matter detectors

J. Amaré, J. Castel, S. Cebrián, I. Coarasa, C. Cuesta, T. Dafni,

J. Galán, E. García, J.G. Garza, F.J. Iguaz, I.G. Irastorza, G. Luzón, M. Martínez, H. Mirallas, M.A. Oliván, Y. Ortigoza,

A. Ortiz de Solórzano, E. Ruiz-Chóliz, J. Puimedón, M.L. Sarsa, J.A. Villar, P. Villar

PII: S0927-6505(17)30189-5

DOI: 10.1016/j.astropartphys.2017.11.004

Reference: ASTPHY 2258

To appear in: Astroparticle Physics

Received date:

20 June 2017

Revised date:

Accepted date:

Please cite this article as: J. Amaré, J. Castel, S. Cebrián, I. Coarasa, C. Cuesta, T. Dafni, J. Galán, E. García, J.G. Garza, F.J. Iguaz, I.G. Irastorza, G. Luzón, M. Martínez, H. Mirallas, M.A. Oliván, Y. Ortigoza, A. Ortiz de Solórzano, E. Ruiz-Chóliz, J. Puimedón, M.L. Sarsa, J.A. Villar, P. Villar, Cosmogenic production of tritium in dark matter detectors, Astroparticle Physics (2017), doi: 10.1016/j.astropartphys.2017.11.004

This is a PDF file of an unedited manuscript that has been accepted for publication. As a service to our customers we are providing this early version of the manuscript. The manuscript will undergo copyediting, typesetting, and review of the resulting proof before it is published in its final form. Please note that during the production process errors may be discovered which could affect the content, and all legal disclaimers that apply to the journal pertain. 


\title{
Cosmogenic production of tritium in dark matter detectors
}

\author{
J. Amaré, J. Castel, S. Cebrián ${ }^{1}$, I. Coarasa, C. Cuesta ${ }^{2}$, T. Dafni,
}

J. Galán³ ${ }^{3}$ E. García, J.G. Garza, F.J. Iguaz ${ }^{4}$, I.G. Irastorza, G. Luzón,

M. Martínez ${ }^{5}$, H. Mirallas, M.A. Oliván, Y. Ortigoza, A. Ortiz de

Solórzano, J. Puimedón, E. Ruiz-Chóliz, M.L. Sarsa, J.A. Villar ${ }^{6}$, P. Villar

Universidad de Zaragoza, Pedro Cerbuna 12, 50009 Zaragoza, Spain

Laboratorio Subterráneo de Canfranc, Paseo de los Ayerbe s/n,22880 Canfranc

Estación, Huesca, Spain

\begin{abstract}
\end{abstract}
The direct detection of dark matter particles requires ultra-low background conditions at energies below a few tens of $\mathrm{keV}$. Radioactive isotopes are produced via cosmogenic activation in detectors and other materials and those isotopes constitute a background source which has to be under control. In particular, tritium is specially relevant due to its decay properties (very low endpoint energy and long half-life) when induced in the detector medium, and because it can be generated in any material as a spallation product. Quantification of cosmogenic production of tritium is not straightforward, neither experimentally nor by calculations. In this work, a method for the calculation of production rates at sea level has been developed and applied to some of the materials typically used as targets in dark matter detectors (germanium, sodium iodide, argon and neon); it is based on a selected de-

\footnotetext{
${ }^{1}$ Corresponding author (scebrian $@$ unizar.es)

2 Present Address: Centro de Investigaciones Energéticas, Medioambientales y Tecnológicas, CIEMAT, 28040 Madrid, Spain

${ }^{3}$ Present address: INPAC and Department of Physics and Astronomy, Shanghai Jiao Tong University, Shanghai Laboratory for Particle Physics and Cosmology, 200240 Shanghai, China

${ }^{4}$ Present address: IRFU, CEA, Université Paris-Saclay, F-91191 Gif-sur-Yvette, France ${ }^{5}$ Present Address: Università di Roma La Sapienza, Piazzale Aldo Moro 5, 00185 Roma, Italy

${ }^{6}$ Deceased
} 
scription of tritium production cross sections over the entire energy range of cosmic nucleons. Results have been compared to available data in the literature, either based on other calculations or from measurements. The obtained tritium production rates, ranging from a few tens to a few hundreds of nuclei per $\mathrm{kg}$ and per day at sea level, point to a significant contribution to the background in dark matter experiments, requiring the application of specific protocols for target material purification, material storing underground and limiting the time the detector is on surface during the building process in order to minimize the exposure to the most dangerous cosmic ray components.

Keywords: Cosmogenic activation, Tritium, Germanium, NaI, Noble gases, Rare events

\section{Introduction}

Many different efforts are being devoted worldwide to the study of dark matter which could be pervading the galactic halo $[1,2]$. One of the strategies followed is the direct detection of Weakly Interacting Massive Particles (WIMPs) proposed to constitute this dark matter, making use of different kinds of very sensitive radiation detectors [3]. The expected counting rates from the interaction of WIMPs are extremely low (of the order of a few events per year and ton of detector/or even below), as it is also the case in the study of other rare phenomena; therefore, dark matter detectors require ultra-low background conditions. Operating in deep underground locations, using active and passive shields and selecting carefully radiopure materials, reduces very efficiently the background for rare events experiments $[4,5]$.

In this context, long-lived radioactive impurities in the materials of the set-up induced by the exposure to cosmic rays at sea level (during fabrication, transport and storage) may be even more important than residual contamination from primordial nuclides and become very problematic, depending on the target. For instance, the poor knowledge of cosmic ray activation in detector materials is highlighted in [6] as one of the three main uncertain nuclear physics aspects of relevance in the direct detection approach pursued to solve the dark matter problem. In principle, cosmogenic activation can be kept under control by minimizing exposure at surface and storing materials underground, avoiding flights and even using shields against the hadronic component of cosmic rays during surface detector building or operation. But since these requirements usually complicate the preparation of experiments 
(for example, while crystal growth and detector mounting steps) it would be desirable to have reliable tools to quantify the real danger of exposing the different materials to cosmic rays. Direct measurements (by sensitive screening of exposed materials) and different calculations of yields have been performed for several materials in the context of dark matter, double beta decay and neutrino experiments [7]. Many different studies are available for germanium [8-16] and interesting results have been derived in the last years also for other detector media like sodium iodide $[17,18]$, tellurium and tellurium oxide [19-21], xenon [22, 23] or neodymium [24, 25] as well as for materials commonly used in the set-ups like copper [14, 22, 23, 26, 27], lead [28], stainless steel [23, 26], titanium [23] and teflon [23].

Spallation of nuclei by high energy nucleons is a very relevant process in cosmogenic activation, but other reactions like fragmentation, induced fission or capture are also important for some nuclei. Tritium is one of radioactive isotopes that can be cosmogenically induced in many materials by several production channels, contributing as a very relevant background in the detector medium of dark matter experiments due to its decay properties: it is a pure beta emitter with transition energy of $18.591 \mathrm{keV}$ and a long half-life of 12.312 y [29]. Following the shape of the beta spectrum for the superallowed transition of ${ }^{3} \mathrm{H}, 57 \%$ of the emitted electrons are in the range from 1 to $7 \mathrm{keV}$; these electrons are typically fully absorbed since most of the dark matter detectors are large enough. Due to the long half-life of tritium, saturation activity is difficult to reach; however, even below saturation, as tritium emissions are concentrated in the energy region where the dark matter signal is expected, tritium can beimportant and it is worth of consideration. Quantification of tritium cosmogenic production is not easy, neither experimentally since its beta emissions are hard to disentangle from other background contributions, nor by calculations, as tritium can be produced by different reaction channels. Some studies on tritium production in materials of interest for dark matter experiments can be found in $[8,12,16,23,30]$; the aim of this work has been to find a reliable method to quantify the production rate of tritium in several detector media used in WIMP direct detection. The production cross sections at the different energies (the so-called excitation functions) have been selected over the entire energy range of cosmic nucleons and the calculations made have been compared with available data.

The following materials have been taken into consideration. Natural isotopic abundances have been assumed unless specifically stated. 
- Germanium is being used as a target for dark matter searches for many years, either as pure ionization detectors [31-33] or in cryogenic detectors measuring simultaneously ionization and heat $[34,35]$. Tritium is highlighted as one of the relevant background sources in future experiments like SuperCDMS [36]. A first calculation of tritium production in germanium was made in [8], followed by others in [12, 23], a part of the unexplained background in the low energy region of the IGEX detectors could be attributed to tritium [37]. Recently, a very detailed quantification of cosmogenic products including tritium has been made by the EDELWEISS collaboration [16] and presence of tritium in the enriched germanium detectors ( $87 \%$ of ${ }^{76} \mathrm{Ge}$ and $13 \%$ of ${ }^{74} \mathrm{Ge}$ ) of the Majorana Demonstrator focused on the study of the double beta decay has been reported too [38]. Therefore, calculations of tritium production in germanium can be cross-checked with all this available information.

- $\mathrm{NaI}(\mathrm{Tl})$ is the scintillator used in the DAMA/LIBRA experiment, which has observed an annual modulation effect in the detection rate with a very high confidence level [39]; other experiments and projects like ANAIS [40], KIMS [41] and DM-ICE [42] (now joint in COSINE) or SABRE [43] are underway in order to confirm this result using the same target. Production of tritium in this material had not been faced, although DAMA/LIBRA experiment was able to produce limits to the presence of this isotope in its detectors [44]. The commissioning of the ANAIS (Annual Modulation with $\mathrm{NaI}(\mathrm{Tl})$ Scintillators) experiment with nine detectors having a total $\mathrm{NaI}(\mathrm{Tl})$ mass of $112.5 \mathrm{~kg}$ is underway in 2017 at the Canfranc Underground Laboratory (Laboratorio Subterráneo de Canfranc, LSC), Spain, but the first modules are fully operative for several years. In the ANAIS detectors, after a detailed analysis of several cosmogenic products [17], the presence of tritium is inferred in order to explain the differences between the measured background and the background models [45]. Because tritium is a relevant background in the region of interest for the annual modulation signal, tritium production in $\mathrm{NaI}$ is further analyzed in this work.

- In the context of the TREX-DM experiment (TPCs for Rare Event eXperiments for Dark Matter) [46], the use of both Ar and Ne gas is envisaged and therefore the production of tritium in these two materi- 
als has been evaluated too. TREX-DM is an approved experiment to be installed at the LSC intended to detect low-mass WIMPs using a Micromegas-based TPC. The most sensitive experiments in the search for dark matter at present are double phase noble liquid-gas detectors [47]; in the case of xenon target, tritium and other non-noble radioisotopes are easily suppressed by specific purification procedures [48] and hence have not been considered in this study.

The structure of the paper is as follows: in Sec. 2 the process of calculating the production rates of tritium based on selected excitation functions is presented; results obtained for the different targets considered are shown and discussed in Sec. 3, comparing with available prefious estimates; finally, conclusions are summarized in Sec. 4.

\section{Calculations}

Cosmogenic activation is strongly dependent on the nucleon flux, neutron to proton ratio, and energies available. At sea level, for instance, the flux of neutrons and protons is virtually the same at energies of a few $\mathrm{GeV}$; however, at lower energies the proton to neutron ratio decreases significantly because of the absorption of charged particles in the atmosphere. For example, at $100 \mathrm{MeV}$ this ratio is about $3 \%$ [49]. Consequently, at the Earth's surface, nuclide production is mainly dominated by neutrons. If materials were flown at high altitude where cosmic flux is much greater, energies at play would be larger and activation by protons should not be neglected. As confirmed by calculations in $[23,50]$, contribution to tritium production by muons is irrelevant. Then, only neutron activation at sea level has been taken into account in this work.

The flux of cosmic rays $(\phi)$ and the production cross-section $(\sigma)$ are the two basic ingredients in the calculation of the production rate $R$ of any isotope by the exposure to a flux of cosmic rays, which can be evaluated as:

$$
R=N \int \sigma(E) \phi(E) d E
$$

being $N$ the number of target nuclei and $E$ the particle energy. For the neutron spectrum at sea level, the parametrization from [51] has been considered (see figure 1). It is based on a set of measurements of cosmic neutrons on the ground across the US, accomplished using Bonner sphere spectrometers [52]; 


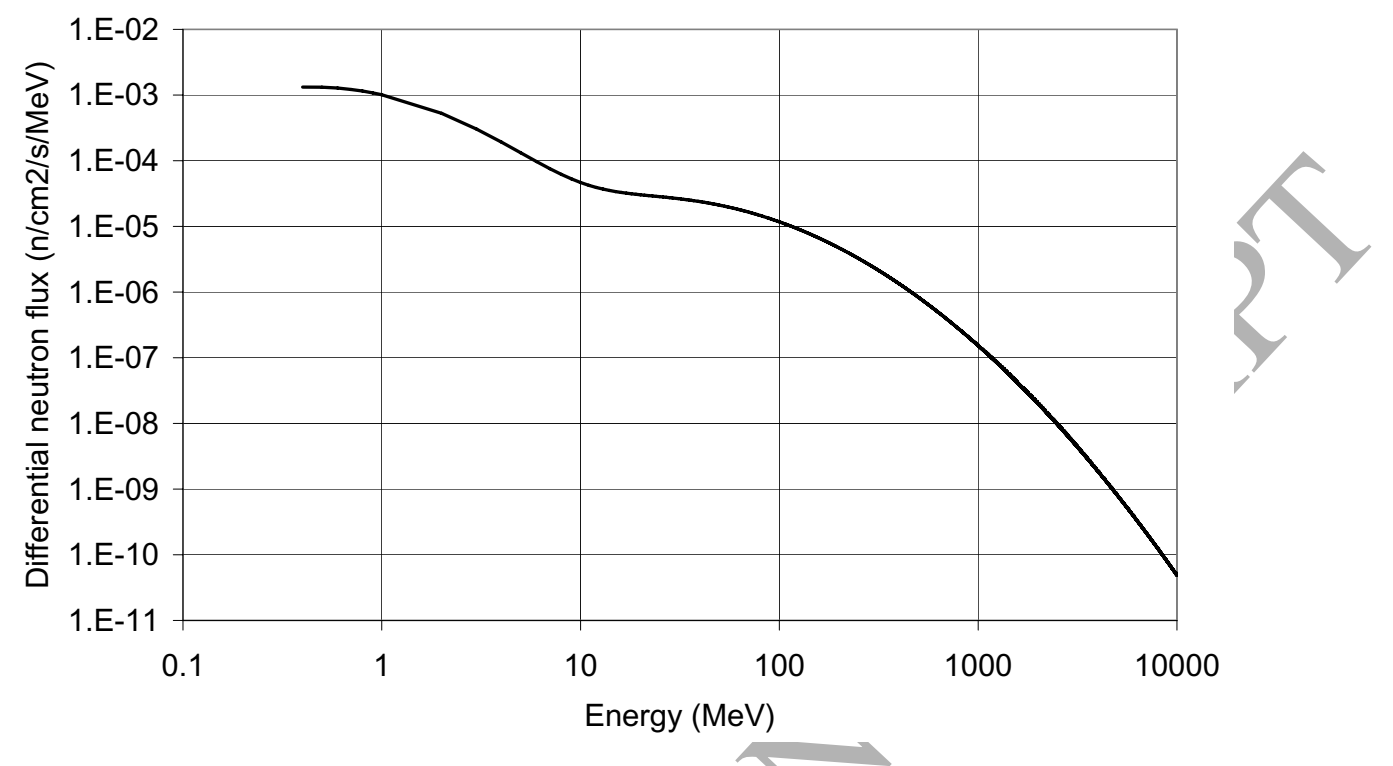

Figure 1: Energy spectrum of cosmic neutrons following the analytic expression in [51] (for conditions of New York City at sea leyel) which has been considered in this work to calculate production rates of cosmogenic isotopes.

an analytic expression fitting data for energies above $0.4 \mathrm{MeV}$ was deduced for reference conditions (New York City, sea level, mid-level solar modulation) [51]. Applying this description of cosmic neutrons the integral flux from $10 \mathrm{MeV}$ to $10 \mathrm{GeV}$ is $3.6 \times 10^{-3} \mathrm{~cm}^{-2} \mathrm{~s}^{-1}$.

Concerning the production cross sections, the methodology proposed in [7] has been followed: first, collect information from different sources of data, taken into account both measurements of production cross sections and calculations using computational codes; then, select the best description of the excitation function by nucleons. The following sources have been considered:

- The EXFOR database (CSISRS in US) [53] provided the very scarce experimental data of tritium production for $\mathrm{Ne}$, Ar and $\mathrm{Na}$, all coming from an irradiation experiment with neutrons having an energy spectrum peaked at $22.5 \mathrm{MeV}$ [54].

- At library TENDL (TALYS-based Evaluated Nuclear Data Library) [55], cross sections obtained with the TALYS nuclear model code system were found for neutrons and protons up to $200 \mathrm{MeV}$ for all the targets. 
TENDL-2013 was used for neutrons and TENDL-2015 for protons ${ }^{7}$.

- Library HEAD-2009 (High Energy Activation Data) [56] merges data for neutrons and protons from $150 \mathrm{MeV}$ to $1 \mathrm{GeV}$. Here, data from HEPAD-2008 (High-Energy Proton Activation Data) sub-library have been considered, obtained using a selection of models and codes (CEM 03.01, CASCADE/INPE, MCNPX 2.6.0, ...) dictated by an extensive comparison with EXFOR data. Only targets with $\mathrm{Z} \geq 12$ are considered, and therefore, neither Ne nor Na data are available in this library.

- The YIELDX routine (implementing the most updated version of the popular Silberberg \& Tsao semiempirical formulae [57-59] giving nucleon - nucleus cross sections for different reactions) allows to compute production cross sections. Although it can be used for a wide range of targets and products at energies above $100 \mathrm{MeV}$, only products as ${ }^{6} \mathrm{He}$ and heavier can be evaluated, and therefore, no information on ${ }^{3} \mathrm{H}$ could be obtained.

Figures 2-5 show all the available information on the excitation function by nucleons collected for the different analyzed targets, together with some extrapolations in the high energy range which will be described in the next section. Independent results for neutrons and protons are presented whenever possible, to compare the corresponding cross sections, even if proton activation has not been evaluated here. Production rates have been computed following equation 1 , by convoluting a particular excitation function with the described energy spectrum of cosmic neutrons at sea level; the energy step in the calculations has been $0.1 \mathrm{keV}$ for TENDL data integration, $1 \mathrm{keV}$ for HEAD-2009 data up to $1 \mathrm{GeV}$ and $100 \mathrm{keV}$ at higher energies.

\section{Results and discussion}

In order to choose the best description of excitation functions, it would be desirable to evaluate deviation factors between calculated and experimental cross sections; for the tritium production considered here, unfortunately, there is only one experimental point for $\mathrm{Ar}, \mathrm{Na}$ and $\mathrm{Ne}$ (see section 2). For

\footnotetext{
${ }^{7}$ The older version was chosen for neutrons due to the availability in the "Tabular production and total cross sections" section of the library of the channel of interest for tritium $(t)$ production for the targets of interest $\left({ }^{A} Y\right):{ }^{A} Y(n, x) t$.
} 

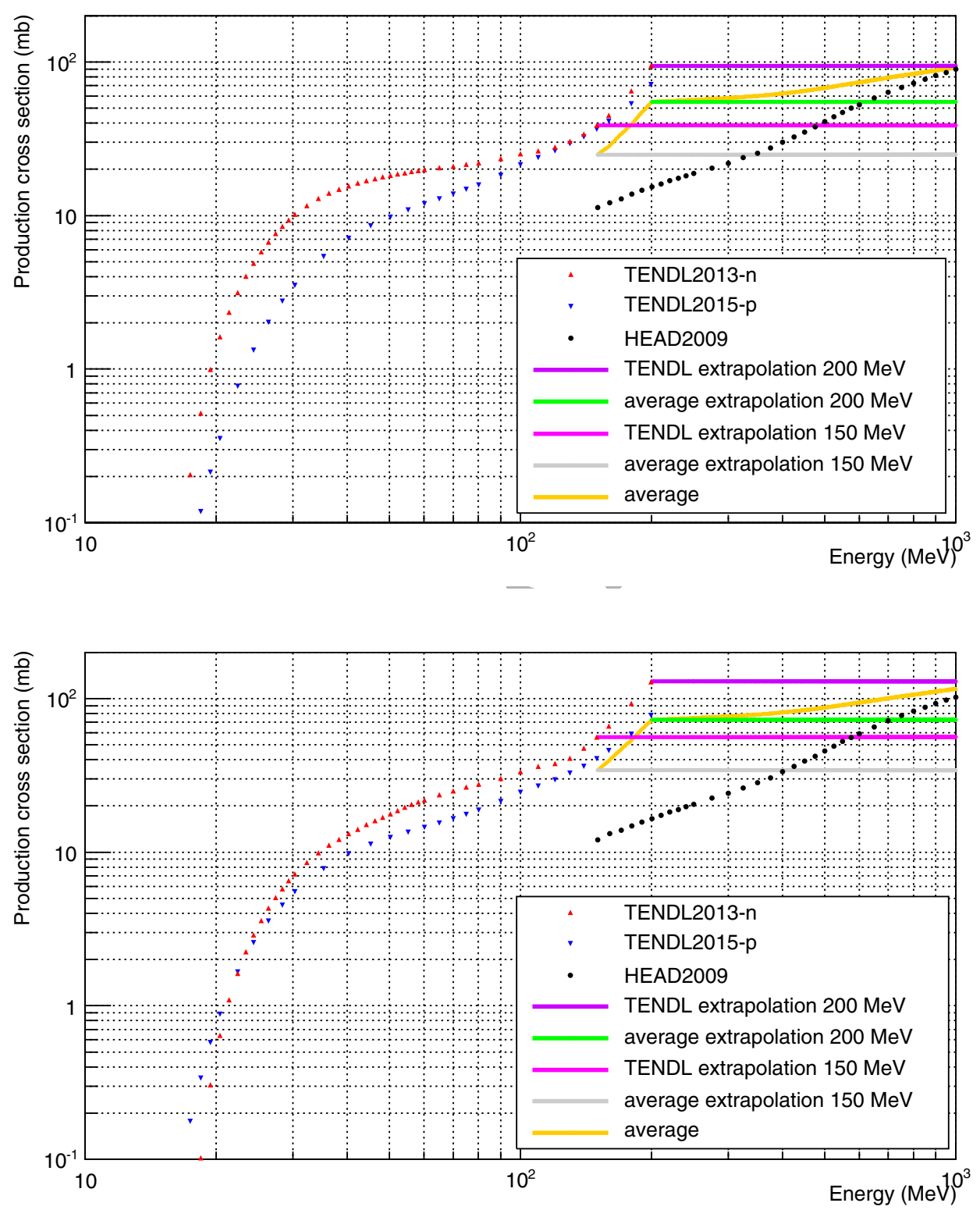

Figure 2: Comparison of excitation functions for the production of ${ }^{3} \mathrm{H}$ on natural (top) and enriched (bottom) Ge by nucleons taken from different sources (TENDL-2013 and HEAD2009 libraries) together with several extrapolations considered at high energies (see text). Above $1 \mathrm{GeV}$ (not shown in the plots), a constant production cross-section from the last available energy has been assumed. An isotopic composition of $87 \%$ for ${ }^{76} \mathrm{Ge}$ and $13 \%$ for ${ }^{74} \mathrm{Ge}$ has been considered for the enriched germanium. 

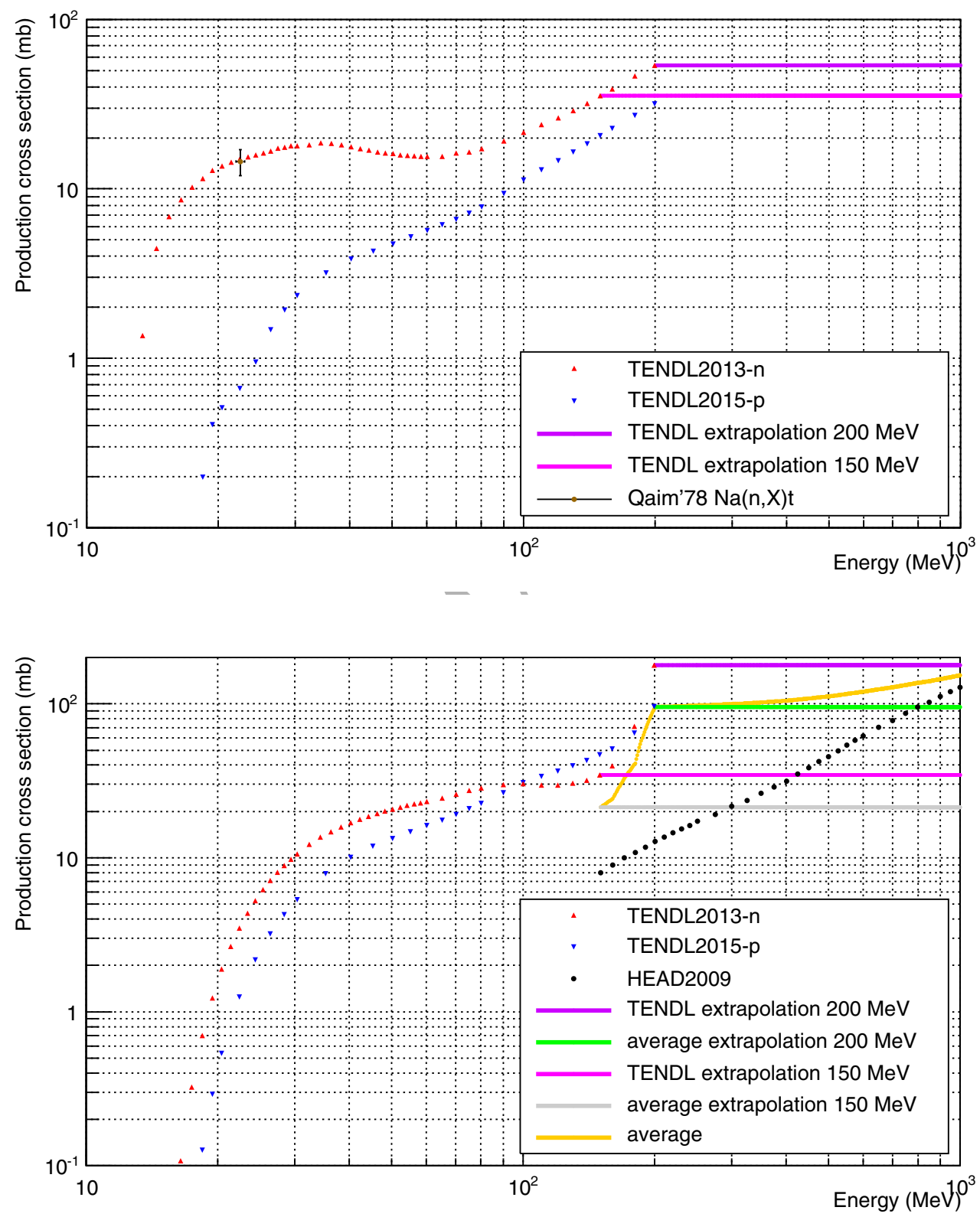

Figure 3: As plots in figure 2, but for $\mathrm{Na}$ (top) and I (bottom) as targets. 


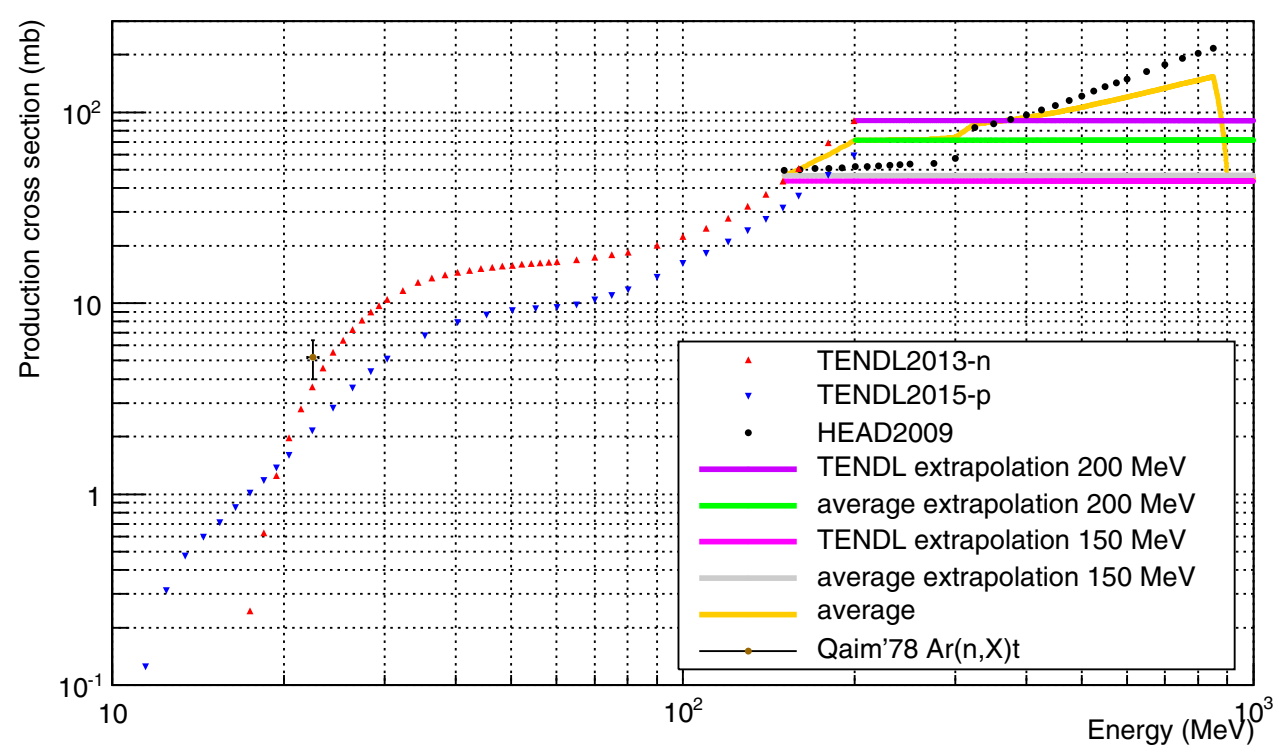

Figure 4: As plots in figure 2, but for Ar as target.

the lowest energies, the TENDL data for neutrons up to $200 \mathrm{MeV}$ reproduce well the measurements in the three cases. At higher energies, as there is no experimental data to validate the cross section selection, and the trend of the cross-section values suggests that the contribution cannot be neglected, different assumptions have been considered. Then, production rates have been derived for different conditions in the calculation of the integral in equation 1 and are shown in table 1 . For the higher energies, above $150 / 200 \mathrm{MeV}$, the evaluated options are the following:

1. HEAD-2009 cross sections have been used, if available. It is assumed that in this high energy range neutron and proton cross sections are comparable.

2. The available highest energy cross-section from TENDL has been considered as a constant value at higher energies.

3. The average between the available highest energy cross-section from TENDL and the corresponding results from HEAD-2009 has been considered also as a constant value at higher energies.

4. The average at each energy between TENDL data (and its extrapola- 


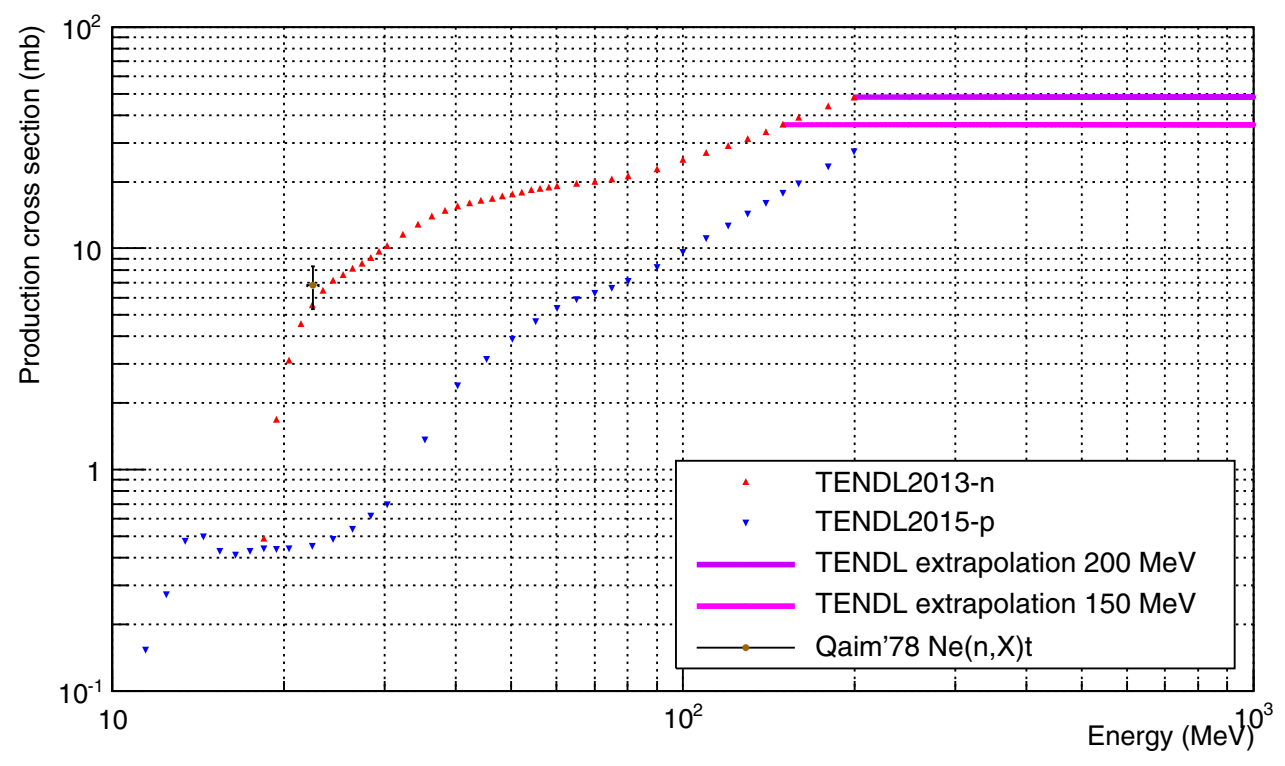

Figure 5: As plots in figure 2, but for Ne as target.

tion) and HEAD-2009 cross sections has been taken into consideration too.

In table 1 and figures 2-5, for brevity sake, option (1) is referred as HEAD2009, option (2) as TENDL extrapolation, option (3) as average extrapolation and option (4) as average.

No data have been found above $1 \mathrm{GeV}$. Although the cosmic neutron spectrum decreases quickly with energy (see figure 1), cross sections seem to be still increasing with energy (see figures 2-5) and then calculations of production rates have been extended up to $10 \mathrm{GeV}$; since is difficult to predict the particular shape of the excitation functions in this energy range, a constant production cross-section from the last available energy has been assumed, giving actually a lower limit to the contribution to the production rates from neutrons of those energies. As it will be shown afterwards, that contribution is estimated to be low enough to make this approximation not critical. 


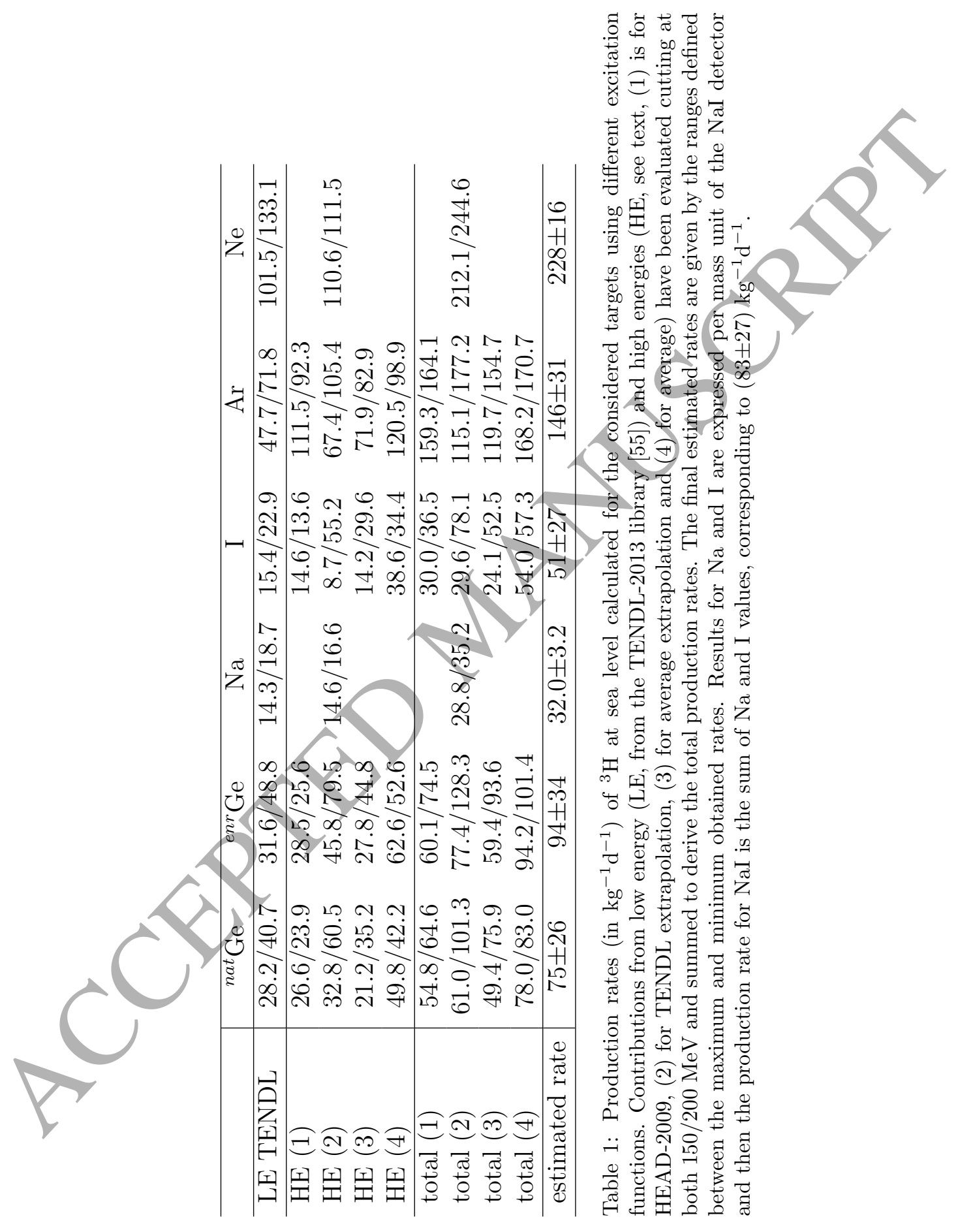


In the range between 150 and $200 \mathrm{MeV}$ there are (at least for some targets) cross sections from both TENDL and HEAD-2009. But unfortunately, there is an important mismatch between excitation functions from the two different libraries used at lower and higher energies, especially for germanium and iodine (see figures 2 and 3). The lack of experimental results on cross sections here makes difficult to choose one description. This introduces an important uncertainty in the estimate of the production rate; to take into account and to quantify this effect, the whole process of calculating the production rate by summing the contribution from low energy (using TENDL) and that from high energy (for each of the described four options) has been made twice, considering the cut between low and high energy either at 150 or)at $200 \mathrm{MeV}$. Table 1 summarizes all the obtained production rates in the eight different conditions. The maximum and minimum rates define an interval, whose central value and half width have been considered as the final results and their uncertainties for the evaluation of the production rates of tritium in the different targets. For NaI, rates derived for $\mathrm{Na}$ and I have been properly summed.

It is worth noting that the actual production rates must be higher than the values reported in table 1 since they correspond only to neutron activation. Proton activation, being much smaller, is not completely negligible. As pointed out in section 2, neutron and proton fluxes are similar on the Earth surface for energies above $1 \mathrm{GeV}$. The relative contribution in the total production rate of neutrons from this very high energy range is for instance between 4 and $7 \%$ for all the analyzed targets when considering cross sections from TENDL extrapolation (total (2) in table 1) and $10 \%$ and $13 \%$ for germanium and iodine respectively when assuming the HEAD-2009 cross sections (total (1) in table 1). Therefore, at least a similar relative contribution would be expected from protons. These percentages agree with the results in $[11,50]$ where proton activation in germanium was specifically evaluated for some isotopes and with the typical contribution from protons to isotope production quoted as $\sim 10 \%$ in [49].

Table 2 compares our estimated rates with the available information on tritium production rates from the literature. Results from [12] were estimated generating the excitation functions with TALYS 1.0 code; TALYS [60] is a software package for the simulation of nuclear reactions that can be used in the $1 \mathrm{keV}$ to $200 \mathrm{MeV}$ incident energy range. Results from [23] are based on GEANT4 simulation or ACTIVIA calculations [61] considering neutrons from thermal energies to $100 \mathrm{GeV}$; for GEANT4 simulations the 


\begin{tabular}{l|ccccc}
\hline & ${ }^{n a t} \mathrm{Ge}$ & ${ }^{e n r} \mathrm{Ge}$ & $\mathrm{NaI}$ & $\mathrm{Ar}$ & $\mathrm{Ne}$ \\
\hline This work & $75 \pm 26$ & $94 \pm 34$ & $83 \pm 27$ & $146 \pm 31$ & $228 \pm 16$ \\
Measurements & $82 \pm 21[16]$ & $140 \pm 10[64]$ & & & \\
& $76 \pm 6[62]$ & & & & \\
TALYS [12] & 27.7 & 24.0 & 31.1 & 44.4 & \\
GEANT4 [23] & 48.3 & & 42.9 & 84.9 & \\
GEANT4 [50] & & 51.3 & & & \\
ACTIVIA [23] & 52.4 & & 36.2 & 82.9 \\
ACTIVIA [50] & & 47.4 & & \\
ACTIVIA & $46 / 43.5[16]$ & & $26[18]$ & \\
COSMO [37] & & 70 & & \\
Ref. [8] & $178 / 210$ & $113 / 140$ & & \\
\hline
\end{tabular}

Table 2: Comparison of the production rates $\left(\mathrm{in}_{\mathrm{kg}}{ }^{-1} \mathrm{~d}^{-1}\right)$ of ${ }^{3} \mathrm{H}$ at sea level evaluated in this work with available information from the literature for the considered targets. The two values from [8] were derived using two different neutron spectra (normalized to sea level at $45^{\circ}$ north latitude) and the two values from ACTIVIA in [16] correspond to using just semiempirical cross sections or data from MENDL-2P too.

set of electromagnetic and hadronic physics processes included in the Shielding modular physics list were taken into account while in ACTIVIA cross sections are obtained from data tables and semiempirical formulae [57-59]. It is worth noting that estimates in $[12,23]$ used the same cosmic neutron spectrum considered in this work from [51] (which required a modification of the ACTIVIA code when using this package), so discrepancies between estimates cannot be assigned to the cosmic ray flux.

A discussion of all the results is given in the following for each one of the considered targets.

\subsection{Germanium}

The first experimental estimate of tritium production rate in natural germanium at sea level has been presented by the EDELWEISS collaboration [16], following a detailed analysis of a long measurement with many germanium detectors; their exposure history above ground during different steps of production and shipment is well-known and has been considered. Another experimental evaluation of the tritium production rate based on CDMSlite data [62] has been presented too, fully compatible with that of EDELWEISS. An estimate of the rate was made using ACTIVIA code and the spectrum from [51] by EDELWEISS [16]. Several calculations had been 
made before: a rough calculation was attempted in [8] using two different neutron spectra; more recent calculations from [12, 23] using different approaches are shown in table 2 together with that presented in this work. All these new calculations summarized in table 2 give lower values than the measured rate; in particular, the smallest value from [12] can be understood because using TALYS cross-sections only contributions from the lowest energy neutrons are considered. The range derived in this work for the production rate and presented in table 1 is well compatible with the measured rates by EDELWEISS and CDMSlite.

For enriched germanium, as used in double beta decay experiments, there are calculations in [8] and using the COSMO code [63] in [37]. In [50], the methodology applied in [23] to evaluate production rates using GEANT4 and ACTIVIA has been used not only for natural ${ }^{8}$ but also to enriched germanium. A first estimate of the production rate from the data of the Majorana Demonstrator, whose enriched detectors have a very wellknown exposure history, has been recently presented [64]; the fitting model to derive the abundance of cosmogenic products is comprised of a calculated tritium beta-decay spectrum, flat background, and multiple X-ray peaks. In this work, the tritium production rate has been evaluated not only for natural but also for enriched germanium. All the results for the enriched material are compared in table 2. According to measured rates and results obtained in this work, production is higher than in natural germanium; this is due to the fact that cross sections increase with the mass number of the germanium isotope in all the energy range above $\sim 50 \mathrm{MeV}$, according to TENDL-2013 and HEAD-2009 data.

\subsection{Sodium lodide}

Only calculations of tritium production rate in $\mathrm{NaI}$ are available; results from $[12,23]$ using different approaches are shown in table 2 together with the estimate from this work. An estimate using ACTIVIA and the spectrum from [51] from $1 \mathrm{MeV}$ to $10 \mathrm{GeV}$ [18] is also presented. As for germanium, the TALYS estimate gives a low rate which is in reasonable agreement with the contribution from just low energy neutrons according to TENDL cross sections derived in this work (see table 1). As shown in figure 3, bottom, in

\footnotetext{
${ }^{8}$ Results on tritium production rates in natural germanium for neutron activation in [50] are virtually the same as those presented in [23] and for this reason they are not reported again in table 2 .
} 
the medium energy range there is a significant difference between HEAD-2009 cross sections and the value extrapolated from TENDL, which is much higher; this fact is responsible of the large dispersion in the estimated production rates from different excitation functions for I. ACTIVIA code is based on semiempirical formulae derived from proton cross sections, as those compiled in HEAD-2009 library; this could be one reason why ACTIVIA estimates give lower production rates than those obtained in this work.

The first ANAIS modules have been taking data at the LSC for several years in order to study the detector response and background. Using data taken with the first two $12.5 \mathrm{~kg} \mathrm{NaI}(\mathrm{Tl})$ detectors produced by the Alpha Spectra company for the ANAIS experiment, production rates of several I and Te isotopes and of ${ }^{22} \mathrm{Na}$ could be derived [17], thanks to the very fast start of data taking after moving the detectors underground. Although a direct identification of a tritium content in the crystals has not been possible, the construction of a detailed background model of these modules and those produced afterwards (based on a Geant4 simulation of quantified background components) points to the need of an additional background source contributing only in the very low energy region, which could be tritium (see details at [45],[65]). The simulated spectra including all well-known contributions agree reasonably with the ones measured, except for the very low energy region; as shown in figure 6 , the inclusion of a certain activity of ${ }^{3} \mathrm{H}$ homogeneously distributed in the NaI crystal provides a very good agreement also below $20 \mathrm{keV}$. Figure 6 compares data and background models for two detectors, named D0 and D2, with different production history. D0 arrived at LSC in December 2012 and D2 in March 2015, and both have been taking data there since then in several set-ups. The shown data correspond to 59.9 days of measurement in September and October 2016. No fitting has been attempted, but the required ${ }^{3} \mathrm{H}$ initial activities (that is, at the moment of going underground) to reproduce the data would be around $0.20 \mathrm{mBq} / \mathrm{kg}$ for D0 and $0,09 \mathrm{mBq} / \mathrm{kg}$ for D2; as explained below, the different values for the two detectors can be understood due to a different time of exposure to cosmic rays for each detector. The value estimated for D2 agrees with the upper limit set on tritium activity for DAMA/LIBRA crystals [44]. Preliminary background models developed for the other ANAIS detectors already operated in Canfranc point to a tritium content similar to that assumed for D2.

Since the exposure history of the NaI material used to produce the crystals (following different procedures for purification and crystal growth) is not 

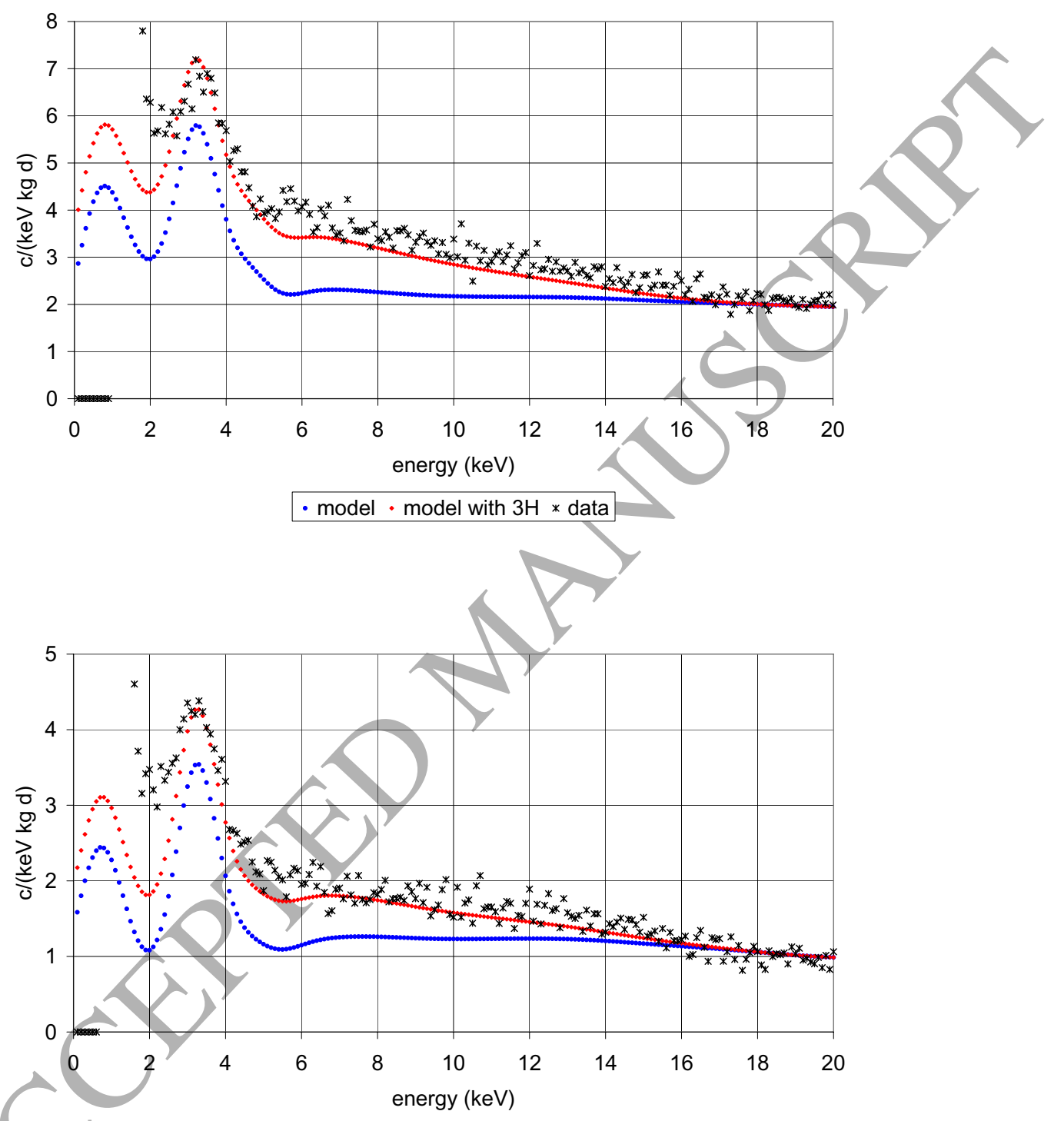

- model $\cdot$ model with $3 \mathrm{H} *$ data

Figure 6: The very low energy region of the energy spectra measured for D0 (top) and D2 (bottom) ANAIS detectors compared with the corresponding simulated models [45] including all the quantified intrinsic and cosmogenic activities in the detectors and main components of the set-up (blue) and adding also tritium in the $\mathrm{NaI}$ crystal (red). $\mathrm{A}^{3} \mathrm{H}$ activity of $0.20 \mathrm{mBq} / \mathrm{kg}$ is considered for $\mathrm{D} 0$ and $0.09 \mathrm{mBq} / \mathrm{kg}$ for $\mathrm{D} 2$. 
precisely known, no attempt of deriving tritium production rates from these estimated activities in ANAIS crystals has been made. But some crosscheck of the results is possible to confirm the plausibility of the tritium hypothesis; assuming that the whole crystal activation took place in Alpha Spectra facilities at Grand Junction, Colorado (where the cosmic neutron flux is estimated to be a factor $f=3.6$ times higher than at sea level [17]) the required exposure time $t_{\text {exp }}$ to produce an activity $A$ of an isotope with decay constant $\lambda$ for a production rate $R$ at sea level can be deduced using

$$
A=f R\left[1-\exp \left(-\lambda t_{\text {exp }}\right)\right]
$$

For the range of production rates estimated in this work (shown in table 1) and the deduced tritium activities in D0 and D2, the exposure times are between 0.8 and 1.6 years and 4.2 and 8.4 months, respectively. These values roughly agree with the time lapse between sodium iodide raw material purification starting and detector shipment, according to the company. As an additional check, for these exposure times, the ratio of the induced initial activities of the also long-living cosmogenic isotope ${ }^{22} \mathrm{Na}$ in D0 and D2 following equation 2 is $\sim 2$, in good agreement with the measured activities

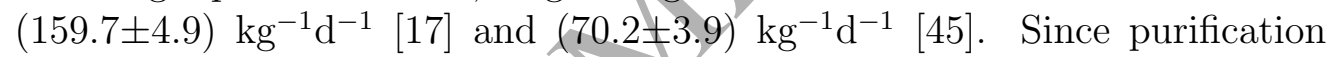
methods cannot remove this isotope, this means that the raw material was not activated before crystal production. Activity of ${ }^{22} \mathrm{Na}$ can be estimated through different distinctive signatures; in particular, once I and Te cosmogenic isotopes have decayed, it can be quantified by measuring the beta emissions absorbed in one detector in coincidence with $1274.5 \mathrm{keV}$ depositions in other one.

\subsection{Argon and Neon}

There is no experimental information of tritium production in argon or neon gas. As shown in table 2, there were estimates of the production rate in argon $[12,23]$ but there was no information for neon. As for other targets, the lowest estimate for argon comes from TALYS due to the limited energy range considered. For argon, the excitation functions from the two different libraries used at low and high energies match reasonably well (see figure 4); therefore, the selected cross-sections seem more reliable than for other targets and the derived production rate has a lower uncertainty.

For TREX-DM experiment, a detailed background model has been built, following the full simulation of the main quantified background sources (thanks 
to the assessment of the radiopurity of all the relevant components) and including the background rejection methods based on the topological information provided by the readout planes [66]. The model predicts a level of $\sim 5$ events $/ \mathrm{keV} / \mathrm{kg} / \mathrm{d}$ in the $2-7 \mathrm{keV}$ region. Tritium emissions are fully absorbed in the gas producing a signal indistinguishable from that of a dark matter interaction. If saturation activity were reached for tritium, according to production rates in table 1 , it would dominate the expected background model with a contribution larger than 10 events $/ \mathrm{keV} / \mathrm{kg} / \mathrm{d}$. However, tritium is expected to be suppressed by purification of gas and minimizing exposure to cosmic rays of the purified gas should avoid any problematic tritium activation. On the other hand, in TREX-DM, mixtures of $\mathrm{Ar}$ ) or Ne with $1-2 \% \mathrm{iC}_{4} \mathrm{H}_{10}$ at $10 \mathrm{~b}$ are foreseen; tritium could not only be cosmogenically induced in the noble gas, but also present in the isobutane No specific information about tritium content in isobutane has been found. Assuming similar concentration as in water ${ }^{9}$, this would also give a very relevant contribution in the RoI of TREX-DM of few tens of events $/ \mathrm{keV} / \mathrm{kg} / \mathrm{d}$ for Ar and Ne. In any case, obtention of isobutane from underground gas sources shielded from cosmic rays will avoid a dangerous tritium content. The first experimental data in TREX-DM, expected for 2018, would be extremely useful to confirm that tritium production is not a relevant background source for the experiment. Further experimental input would help to validate or improve the approach followed in this work.

\section{Conclusions}

The production of tritium due to the exposure to cosmic rays at sea level has been studied for materials used in dark matter detectors, since, as shown by several experimental results, it can become a very relevant background source due to its very low energy beta emissions and long half-life. Production rates have been calculated for germanium, sodium iodide, argon and neon taking into account the neutron spectrum from [51] and selected excitation functions.

In the low energy region below $100 \mathrm{MeV}$, for all the targets, production cross-sections for neutrons are larger than for protons, which confirms the

${ }^{9}$ For natural surface waters there are about one tritium atom per $10^{18}$ atoms of hydrogen, following Ref. [67]. The measured tritium activity in some waters and the limits for drinking water give indeed higher concentrations. 
need to use data specific for neutrons in this energy range. Here, all available neutron data up to $200 \mathrm{MeV}$ have been taken into consideration. The experimental data for cross-sections found, even if scarce, validate the TENDL2013 results considered. For all the targets, cross-sections below $200 \mathrm{MeV}$ increase with energy and therefore contribution from energies above cannot be neglected. Assuming that for energies of a few hundreds of MeV yields by neutrons and protons are similar, cross-sections from HEAD-2009 library can be used; there is in some cases (germanium and iodine) an important mismatch between cross-section from the two libraries, which produces an important uncertainty in the calculation of production rates which has been evaluated. For light targets for which there is no HEAD-2009 result, contribution above $200 \mathrm{MeV}$ has been evaluated assuming as a constant value the available highest energy cross-section.

The tritium production rates estimated in this work, summarized in table 1, are higher than those obtained in previous calculations based on TALYS [12] because of the limited energy range of this code, but also higher than results from GEANT4 and ACTIVIA calculations [23]. It is worth noting that all these estimates are based on the same cosmic neutron spectrum. If no special precautions against tritium production were taken, according to the estimates presented in this work, tritium reaching saturation in germanium, NaI, Ar or Ne detectors would imply in the region from 1 to $7 \mathrm{keV}$ a raw background rate of $7.2,7.9,13.9$, and 21.7 events $/ \mathrm{keV} / \mathrm{kg} /$ day, respectively.

The experimental determination of activated tritium in materials is difficult as it requires low energy threshold detectors and a very good knowledge of other background components to try to identify tritium emissions. The measured production rate in natural germanium by the EDELWEISS collaboration [16] is compatible with the estimated rate here; for enriched germanium, the agreement with the measured rate from the MAJORANA DEmonstrator data is worse but reasonable. Concerning NaI, there are hints of the presence of tritium in the ANAIS detectors [45]; the calculated production rate is compatible with the required exposure times of crystals to produce the estimated possible activities. The acceptable agreement of calculated production rates with available experimental results can be considered as a validation of the method, followed to evaluate tritium yields in other targets as argon or neon and that could be applied also for different materials. 


\section{Acknowledgements}

Professor J.A. Villar passed away in August, 2017. Deeply in sorrow, we all thank his dedicated work and kindness. We acknowledge Yu. A. Korovin for providing the HEAD-2009 library and LSC and GIFNA staff for their support. This work has been financially supported by the Spanish Ministerio de Economía y Competitividad and the European Regional Development Fund (MINECO-FEDER) under grants No. FPA2014-55986-P and FPA2016-76978-C3-1-P; the Consolider-Ingenio 2010 Programme under grants MULTIDARK CSD2009-00064 17 and CPAN CSD2007-00042; from the European Commission under the European Research Council T-REX Starting Grant ref. ERC-2009-StG-240054 of the IDEAS program of the 7th EU Framework Program; and the Gobierno de Aragón and the European Social Fund (Group in Nuclear and Astroparticle Physics).

[1] L. Baudis, Dark matter detection, J. Phys. G: Nucl. Part. Phys. 43 (2016) 044001.

[2] M. Klasen, M. Pohl and G. Sigl, Indirect and direct search for dark matter, Prog. Part. Nucl. Phys. 85 (2015) 1-32.

[3] T. Marrodan-Undagoitia and L, Rauch, Dark matter direct-detection experiments, J. Phys G: Nucl. Part. Phys. 43 (2016) 013001.

[4] G. Heusser, Low-radioactivity background techniques, Annu. Rev. Nucl. Part. Sci. 45 (1995) 543.

[5] J.A. Formaggio and C.J. Martoff, Backgrounds to sensitive experiments underground, Annu. Rev. Nucl. Part. Sci. 54 (2004) 361.

[6] P. Gondolo, Wanted! Nuclear Data for Dark Matter Astrophysics, Nuclear Data Sheet 120 (2014) 175-179.

(7] S. Cebrián, Cosmogenic activation of materials, Int. J. Mod. Phys. A 32 (2017) 1743006.

[8] F. T. Avignone et al., Theoretical and experimental investigation of cosmogenic radioisotope production in germanium, Nucl. Phys. B (Proc. Suppl.) 28A (1992) 280. 
[9] H. Miley et al., New techniques and results in ${ }^{76}$ Ge double-beta decay, Nucl. Phys. B (Proc. Suppl.) 28A (1992) 212.

[10] E. B. Norman et al., Cosmic-Ray Production of Co-60 in Double BetaDecay Source Materials, Nucl. Phys. B (Proc. Suppl.) 143 (2005) 508.

[11] I. Barabanov et al., Cosmogenic activation of germanium and its reduction for low background experiments, Nucl. Instrum. Meth. B 251 (2006) 115-120.

[12] D. M. Mei et al., Cosmogenic production as a background in searching for Rare Physics processes, Astropart. Phys. 31 (2009) 417-420.

[13] S. R. Elliott et al., Fast-neutron activation of long-lived isotopes in enriched Ge, Phys. Rev. C 82 (2010) 054610.

[14] S. Cebrián et al., Cosmogenic activation in germanium and copper for rare event searches, Astropart. Phys. 33 (2010) 316-329.

[15] S. Jian et al., A background simulation method for cosmogenic nuclides inside HPGe detectors for rare event experiments, Nucl. Instrum. Meth. A 763 (2014) 364-371.

[16] E. Armengaud et al,. Measurement of the cosmogenic activation of germanium detectors in EDELWEISS-III, Astropart. Phys. 91 (2017) 5164 .

[17] J. Amaré et al, Cosmogenic radionuclide production in $\mathrm{NaI}(\mathrm{Tl})$ crystals, J. Cosm.Astrop. Phys. 02 (2015) 046.

[18] W.C. Pettus, Cosmogenic Activation in NaI Detectors for Dark Matter Searches, PhD Dissertation, University of Wisconsin-Madison, 2015.

[19] A. F. Barghouty et al., Measurements of p-induced radionuclide production cross sections to evaluate cosmic-ray activation of Te, Nucl. Instrum. Meth. B 295 (2013) 16-21.

[20] V. Lozza and J. Petzoldt, Cosmogenic activation of a natural tellurium target, Astropart. Phys. 61 (2015) 62-71. 
[21] B.S. Wang et al., Cosmogenic-neutron activation of $\mathrm{TeO}_{2}$ and implications for neutrinoless double-beta decay experiments, Phys. Rev. C 92 (2015) 024620.

[22] L. Baudis et al., Cosmogenic activation of xenon and copper, Eur. Phys. J. C 75 (2015) 485.

[23] C. Zhang et al., Cosmogenic activation of materials used in rare event search experiments, Astropart. Phys. 84 (2016) 62-69.

[24] O. Lebeda et al., Excitation functions of proton-induced reactions on natural $\mathrm{Nd}$ in the 10-30 MeV energy range, and production of radionuclides relevant for double-beta decay, Phys. Rev. C 85 (2012) 014602.

[25] O. Lebeda et al., Excitation functions of proton-induced reactions on natural $\mathrm{Nd}$ and production of radionuclides relevant for double beta decay: Completing measurement in 5-35 MeV energy range, Nucl. Phys. A 929 (2014) 129-142.

[26] M. Laubenstein, G. Heusser, Cosmogenic radionuclides in metals as indicator for sea level exposure history, App. Rad. Isot. 67 (2009) 750-754.

[27] I. Coarasa et al., Cosmogenic and primordial radioisotopes in copper bricks shortly exposed to cosmic rays, Journal of Physics: Conference Series $\mathbf{7 1 8}$ (2016) 042049.

[28] V.E. Guiseppe et al., Fast-neutron activation of long-lived nuclides in natural Pb, Astropart. Phys. 64 (2015) 34-39.

[29] Decay Data Evaluation Projet, http://www.nucleide.org/DDEP_WG/DDEPdata.htm.

[30] J. Amaré et al., Production and relevance of cosmogenic radionuclides in NaI(TI) crystals, AIP Conference Proceedings 1672 (2015) 140001.

[31] A, Morales et al., Improved constraints on WIMPs from the international germanium experiment IGEX, Phys. Lett. B 532 (2002) 8.

[32] C.E. Aalseth et al., Search for an Annual Modulation in a p-type Point Contact Germanium Dark Matter Detector, Phys. Rev. Lett. 107 (2011) 141301. 
[33] W. Zhao et al., A Search of Low-Mass WIMPs with p-type Point Contact Germanium Detector in the CDEX-1 Experiment, Phys. Rev. D 93 (2016) 092003.

[34] R. Agnese et al., New Results from the Search for Low-Mass Weakly Interacting Massive Particles with the CDMS Low Ionization Threshold Experiment, Phys. Rev. Lett. 116 (2016) 071301.

[35] E. Armengaud et al., Constraints on low-mass WIMPs from the EDELWEISS-III dark matter search, J. Cosm. Astrop. Phys. 05 (2016) 019 .

[36] R. Agnese et al., Projected sensitivity of the SuperCDMS SNOLAB experiment, Phys. Rev. D 95 (2017) 082002.

[37] S. Cebrián et al., Status of the non-cryogenic dark matter searches at the Canfranc Underground Laboratory, Nucl. Phys. B (Proc. Suppl.) 138 (2005) 147-149.

[38] N. Abgrall et al., New Limits on Bosonic Dark Matter, Solar Axions, Pauli Exclusion Principle Violation, and Electron Decay from the MAJORANA DEMONSTRATOR, Phys. Rev. Lett. 118 (2017) 161801.

[39] R. Bernabei et al., Final model independent result of DAMA/LIBRAphase 1, Eur. Phys. J. C 73 (2013) 2648.

[40] J. Amaré et al., From ANAIS-25 towards ANAIS-250, Physics Procedia $61(2015) 157$.

[41] G. Adhikari et al., Understanding $\mathrm{NaI}(\mathrm{Tl})$ crystal background for dark matter searches, Eur. Phys. J. C 77 (2017) 437.

[42] E. Barbosa de Souza et al., First Search for a Dark Matter Annual Modulation Signal with $\mathrm{NaI}(\mathrm{Tl})$ in the Southern Hemisphere by DMIce17, Phy. Rev. D 95 (2017) 032006.

[43] E. Shields et al., SABRE: A new $\mathrm{NaI}(\mathrm{Tl})$ dark matter direct detection experiment, Physics Procedia 61 (2015) 169.

[44] R. Bernabei et al., The DAMA/LIBRA apparatus, Nucl. Instrum. Meth. A 592 (2008) 297. 
[45] J. Amaré et al., Assessment of backgrounds of the ANAIS experiment for dark matter direct detection, Eur. Phys. J. C 76 (2016) 429.

[46] I.G. Irastorza et al., Gaseous time projection chambers for rare event detection: results from the T-REX project. II. Dark matter, J. Cosm. Astrop. Phys. 01 (2016) 034.

[47] R. Bernabei et al., Liquid noble gases for dark matter searches: An updated survey, Int. J. Mod. Phys. A 301530053 (2015)

[48] D.S. Akerib et al., Radiogenic and muon-induced backgrourds in the LUX dark matter detector, Astropart. Phys. 62 (2015) 33-46.

[49] D. Lal and B. Peters, Cosmic ray produced radioactivity on the Earth, Springer, Berlin-Heidelberg, 1967.

[50] W.Z. Wei et al., Cosmogenic Activation of Germanium Used for TonneScale Rare Event Search Experiments, arXiv:1706.05324v1 [nucl-ex].

[51] M. S. Gordon et al, Measurement of the Flux and Energy Spectrum of Cosmic-Ray Induced Neutrons on the Ground, IEEE Trans. Nucl. Sci. 51 (2004) 3427-3434. Erratum: M.S. Gordon et al., IEEE Transactions on Nuclear Science $\mathbf{5 2}$ (2005) 2703.

[52] P. Goldhagen et al, Measurement of the energy spectrum of cosmic-ray induced neutrons aboard an ER-2 high-altitude airplane, Nucl. Instrum. Meth. A 476 (2002) 42-51.

[53] Experimental Y Nuclear Reaction Data (EXFOR) http://www.nndc.bnl.gov/exfor/exfor.htm, http://wwwnds,iaea.org/exfor/exfor.htm.

[54] S.M. Qaim, R. Wolfle, Triton emission in the interactions of fast neutrons with nuclei, Nucl. Phys. A 295 (1978) 150-162.

[55] A. J. Koning and D. Rochman, Modern Nuclear Data Evaluation With The TALYS Code System, Nuclear Data Sheets 113 (2012) 2841.

[56] Y. A. Korovin et al, High Energy Activation Data Library (HEAD2009), Nucl. Instrum. Meth. A 624 (2010) 20-26. 
[57] R. Silberberg and C. H. Tsao, Astrophys. J. Suppl. Ser. 25 (1973) 315; ibid p. 335.

[58] R. Silberberg and C. H. Tsao, Astrophys. J. Suppl. Ser. 35 (1977) 129; Astrophys. J. Suppl. Ser. 58 (1985) 873; Phys. Rep. 191 (1990) 351.

[59] R. Silberberg and C. H. Tsao, Astrophys. J. 501 (1998) 911.

[60] TALYS, http://www.talys.eu.

[61] J.J. Back, Y.A. Ramachers, ACTIVIA: Calculation of isotope production cross-sections and yields, Nucl. Instrum. Meth. A 586 (2008) 286294.

[62] E. Fascione and W. Rau, Cosmogenic Background in CDMSlite, Poster at the XV International Conference on Topics in Astroparticle and Underground Physics (TAUP 2017), Sudbury, Canada, https://indico.cern.ch/event/606690/contributions/2591554/.

[63] J. Martoff and P.D. Lewin, COSMO- a program to estimate spallation radioactivity produced in a pure substance by exposure to cosmicradiation on the Earth, Comput. Phys. Commun. 72 (1992) 96.

[64] B. White et al., Cosmogenic Induced Backgrounds for the MAJORANA DEMONSTRATOR Experiment, Presentation at Low Radioactivity Techniques 2017 workshop, Seoul, Korea, http://indico.ibs.re.kr/event/46/session/7/contribution/13.

[65] P. Villar, Background evaluation of the ANAIS dark matter experiment in different configurations: towards a final design, $>$ PhD Dissertation, Universidad de Zaragoza, 2016, https://zaguan.unizar.es/record/58561/files/TESIS- 2017-007.pdf.

[66] F.J. Iguaz et al., TREX-DM: a low-background Micromegas-based TPC for low-mass WIMP detection, Eur. Phys. J. C 76 (2016) 529.

[67] D.G. Jacobs, Sources of tritium and its behaviour upon release to the environment, AEC Critical Review Series, 1968. 